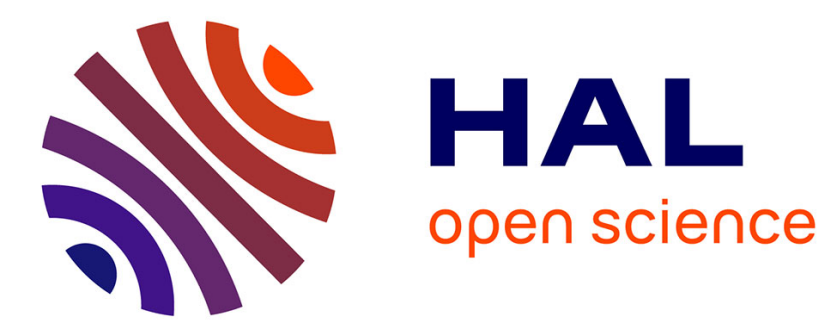

\title{
On the law relating processing to storage in working memory.
}

Pierre Barrouillet, Sophie Portrat, Valérie Camos

\section{To cite this version:}

Pierre Barrouillet, Sophie Portrat, Valérie Camos. On the law relating processing to storage in working memory.. Psychological Review, 2011, 118 (2), pp.175-92. 10.1037/a0022324 . hal-00824083

\section{HAL Id: hal-00824083 \\ https://hal.science/hal-00824083}

Submitted on 21 May 2013

HAL is a multi-disciplinary open access archive for the deposit and dissemination of scientific research documents, whether they are published or not. The documents may come from teaching and research institutions in France or abroad, or from public or private research centers.
L'archive ouverte pluridisciplinaire HAL, est destinée au dépôt et à la diffusion de documents scientifiques de niveau recherche, publiés ou non, émanant des établissements d'enseignement et de recherche français ou étrangers, des laboratoires publics ou privés. 
Psychological Review, In press

\title{
On the law relating processing to storage in working memory
}

\author{
Pierre Barrouillet \\ Université de Genève \\ Sophie Portrat \\ Université de Genève \\ Valérie Camos \\ Université de Bourgogne et Institut Universitaire de France
}

Running head: Processing and storage in working memory

\section{Corresponding author:}

Pierre Barrouillet

Université de Genève

Faculté de Psychologie et des Sciences de l'Education

40 bd du Pont D'arve

1211 Genève 4 Switzerland

Tel: 41223799251

Pierre.Barrouillet@unige.ch 


\begin{abstract}
Working memory is usually defined in cognitive psychology as a system devoted to the simultaneous processing and maintenance of information. However, though many models of working memory have been put forward during the last decades, they often leave underspecified the dynamic interplay between processing and storage. Moreover, the account of their interaction proposed by the most popular Baddeley and Hitch's multiple-component model is contradicted by facts, leaving unresolved one of the main issues of cognitive functioning. In this article, we derive from the Time-Based Resource-Sharing model of working memory a mathematical function relating the cognitive load involved by concurrent processing to the amount of information that can be simultaneously maintained active in working memory. A meta-analysis from several experiments testing the effects of processing on storage corroborates the parameters of the predicted function, suggesting that it properly reflects the law relating the two functions of working memory.
\end{abstract}




\section{On the law relating processing to storage in working memory}

The main strength of Baddeley and Hitch's (1974) seminal work was undoubtedly to go beyond the unitary short-term store described by Atkinson and Shiffrin (1968) and to set the theoretical basis for a working memory conceived as a system devoted to the simultaneous maintenance and processing of information involved in complex cognitive activities. Accordingly, besides slave systems devoted to store information in the short term, Baddeley and Hitch proposed the existence of a central executive responsible for the manipulation of this information, thus leading to the well known multiple-component model of working memory. However, though this central executive was initially conceived as having also some capacity of storage, the further versions of the theory rapidly restricted its role to processing (Baddeley, 1986), thus delineating a strict structural distinction between the two functions. As a result, the multiple-component model considers processing and storage as independent from each other and fueled by specific resources. Unfortunately, as we will see, this assumption of independence has been repeatedly contradicted by facts, suggesting that the multiplecomponent model, which has became the modal theory of working memory, provides an inadequate account of the main role of working memory. More surprisingly, though many models of working memory have subsequently been proposed (e.g., Cowan, 2005; Engle, Kane, \& Tuholski, 1999; Ericsson \& Kintsch, 1995), they have often left underspecified the dynamics of the relationships between processing and storage. This is even more surprising that, since Baddeley and Hitch's (1974) initial inquiry, working memory was primarily introduced as an interface capable of storing and processing information, as opposed to shortterm memory only having storage capacity. The aim of this article is to fill this gap by establishing the law relating processing to storage in working memory. 
This article is organized in the following way. First, we show that the conception of the relationships between processing and storage proposed by the multiple-component theory of working memory is at odds with several recent findings, most of them issuing from studies conducted within the theoretical framework provided by our Time-Based Resource-Sharing model of working memory (TBRS, Barrouillet, Bernardin, \& Camos, 2004). We derive from our theory the mathematical function governing the trade-off between the amount of information that can be maintained active in working memory and the cognitive load involved by concurrent processing, and we show that this mathematical function is corroborated by empirical results through a meta-analysis including previous and new studies in adults. Finally, we show that our theory can account for the seminal findings that led Baddeley and Hitch (1974) to dissociate processing from storage, and we address recent sets of data that have been interpreted as evidence against the existence of a trade-off between the two functions, the hypothesis of a time-related decay in short-term memory, and against the TBRS model itself. In the discussion, we assess the capacity of previous theories of working memory to accommodate the facts reported and to account for the law relating processing to storage.

\section{Processing and storage within the multiple-component model}

The question of the relationships between processing and storage has been at the heart of working memory studies from their very beginning and motivated the seminal study by Baddeley and Hitch (1974). Their aim was to test the modal model of Atkinson and Shiffrin (1968). They reasoned that if short-term memory functions as a working memory, then processing should be dramatically impaired when the capacity of this short-term memory is exhausted by maintenance requirements. Actually, Baddeley and Hitch (1974) observed in several experiments that short term maintenance did not dramatically impair concurrent processing. They concluded that processing and storage share some common resource or 
supply, but also that they are in many respects independent and supported by distinct structures and mechanisms. This was the foundation of the multiple-component model in which slave systems would be in charge of storage, with a phonological loop for verbal information and a visual scratchpad for visuo-spatial information, while a central executive would coordinate their functioning, select appropriate strategies, and control processing. It is worth noting that the original model of Baddeley and Hitch (1974) conceived the central executive with the capacity to store information when needed and thus to supplement the slave systems. However, this latter assumption of a central executive as a general workspace with storage capacity was subsequently abandoned (Baddeley, 1986). Accordingly, it was argued that even a demanding storage could have virtually no impact on complex tasks requiring the central executive such as arithmetic verification or sentence comprehension, while demanding verification or reading would not impair concurrent maintenance (Logie \& Duff, 1996; see also Duff \& Logie, 2001). Thus, the multiple-component approach has clearly separated storage from processing functions that would be supported by different structures and fueled by separate pools of resource (Baddeley \& Logie, 1999).

This clear distinction has been recently blurred by the addition of a new storage component, the episodic buffer. According to Baddeley (2001), this buffer is assumed to be a limited-capacity storage system with the capacity to integrate and bind information from a variety of subsystems into a form of temporary representation. The underlying processing for this episodic memory would be carried out by the central executive that permits access to the episodic buffer through the medium of conscious awareness, influences its content through attention focusing on a given source of information, and binds together information provided by various sources (Baddeley, 2000). It is important to note that, within this new elaboration of the model, the role of the central executive is not restricted to processing. Contrary to the slave systems that have separate rehearsal systems independent of the central executive, the 
maintenance of information within the episodic buffer depends on the central executive through attention (Repovs \& Baddeley, 2006). Thus, it could be assumed that the central executive plays a major role in the storage function of working memory, but things are not so clear. Indeed, Repovs and Baddeley (2006) pointed out that simple representation and maintenance may be independent of the central executive, unless it requires complex binding and integration of information. However, it seems difficult to establish what kind of memory requires complex binding and the notion of "simple" representation is rather vague. For example, Allen, Baddeley, and Hitch (2006) established that binding visual features to represent objects is not complex and could be automatic. Consequently, Allen, Hitch, and Baddeley (2009) proposed to think of the episodic buffer as a passive store fed by a range of sources.

In summary, the introduction of the episodic buffer constitutes a major theoretical advance in accounting for the integration and binding of information in working memory, but it leaves largely unchanged the assumptions of the multiple-component model concerning the independence of the functions of processing and storage. As we will see in the next section, this assumption of independence does not resist a systematic empirical enquiry.

\section{Assessing the independence assumption}

Our own attempt to address the question of the relationships between processing and storage led us to propose the Time-Based Resource-Sharing (TBRS) model of working memory. In line with a well established view that conceives working memory capacity as a general pool of cognitive resources shared between the two functions of processing and storage (Case, 1985; Daneman \& Carpenter, 1980; Just \& Carpenter, 1992; Turner \& Engle, 1989), the main proposal of the TBRS model (Barrouillet et al., 2004) was that maintenance and processing of information in working memory are not independent, but rely on the same limited resource, which is attention. Maintenance would rely on a process of activation and 
reactivation of memory traces through attentional focusing, while goal-directed processing would engage the construction, selection and transformation of representations through attention. Attention is thus a common resource to be shared between processing and storage. Following Pashler's (1998) conception of a central bottleneck and Oberauer's $(2002,2005)$ hypothesis that the focus of attention can select only one element of knowledge at a time as the object of the next cognitive operation, we assumed that only one elementary cognitive step could take place at a time. Thus, when attention is needed for some processing episode, it is not available for the maintenance of memory items. Because the activation of these items suffers from a time-related decay as soon as attention is switched away, memory traces of the to-be-remembered items fade away when attention is occupied by a processing step. A refreshment of these items is thus needed before their complete disappearance through reactivation by attentional focusing. This sharing of attention would be permitted by a rapid and incessant switching of attention from processing to maintenance occurring during short pauses that would be freed while concurrent processing is running.

Subsequently, Barrouillet, Bernardin, Portrat, Vergauwe, and Camos (2007) extended this model and suggested that the mechanisms responsible for both the maintenance and the processing functions of working memory are executive processes that can be gathered together in a central executive whose role is to form, maintain and transform the temporary representations held in working memory. These representations that integrate information from a variety of sources were presented as similar to those maintained within the episodic buffer in Baddeley's model. Following Cowan (2005), we suggested that this integration would result from binding activated features from long-term memory within the focus of attention, executive functions being processes that retrieve, select, and combine the features that form working memory representations by directing attention towards the items of knowledge that are relevant for the task in hand. 
Thus, contrary to the multiple-component model, the TBRS model assumes a complete dependence and a trade-off between processing and storage. Because memory traces fade away when attention is switched away, processing that occupies attention almost continuously should have a highly detrimental effect on concurrent maintenance by preventing refreshing activities to take place. By contrast, processing that allows frequent and long episodes during which attention is available to refresh memory traces should have a weaker impact on concurrent maintenance. As a consequence, we defined cognitive load $(C L)$ as the proportion of time during which a given task occupies attention, thus preventing maintenance of memory traces:

$$
C L=t_{a} / T
$$

where $t_{a}$ is the time during which attention is occupied and $T$ the total time allowed to perform the task. A remarkable aspect of the model is that the impact of processing on maintenance is more dependent on the cognitive load this processing involves than on its nature, even if the TBRS model also allows for some forgetting induced by representation-based interference (Barrouillet et al., 2007).

We tested this model in working memory span tasks that require the maintenance of memory items while performing a concurrent processing activity that involves attention. We predicted that recall performance (e.g., working memory span) should be strongly affected by this concurrent processing, and more precisely by the cognitive load it involves. For this purpose, we designed computer-paced working memory span tasks that allow to control temporal parameters, such as the reading digit span task in which participants have to remember series of letters while reading series of digits presented successively at a fixed rhythm after each letter. Cognitive load was manipulated by varying the number of digits to be read within the interletter intervals as well as the duration of these intervals. It turned out that recall performance was a function of the ratio between the number of digits to be read 
during each interletter interval and the time allowed to read them (Barrouillet et al., 2004). We argued that this ratio reflects the cognitive load involved by the reading digit task. Recall performance decreased as this ratio increased, either by increasing the number of digits to be read or by decreasing the time allowed to read them or both. In the same way, we demonstrated that varying the cognitive load involved in solving elementary one-digit operations, or in assessing the parity of digits had an effect on maintenance of letters, while reading letters at different paces had an effect on the concurrent maintenance of digits (Barrouillet et al., 2004; Gavens \& Barrouillet, 2004; Lépine, Bernardin, \& Barrouillet, 2005)

More recently, the time-related effects of processing on storage were extended to the visuo-spatial domain. Vergauwe, Barrouillet, and Camos (2009) used computer-paced complex span tasks in which visual (series of matrix patterns) and spatial storage (series of ball movements) were combined with both visual (color discrimination) and spatial (symmetry judgments) processing components. As observed in the verbal domain, manipulating the cognitive load involved by these processing components, either by increasing the number of items to be processed or by decreasing the time allowed to process them, had a direct impact on recall performance. Interestingly, and contrary to the domainbased fractionation of visuo-spatial working memory proposed by Logie (1995) or Klauer and Zhao (2004), but in line with the TBRS model, increasing the cognitive load of either the visual or the spatial processing had the same effect on the visual storage, the same being true for the spatial storage.

The findings reported above rule out a strong version of the multiple-component model. According to this version, the slave systems are literally stores exclusively responsible for storage of domain specific information and are never involved in any processing activity, which all resort to a central executive that is itself never involved in storage (Vergauwe, Barrouillet, \& Camos, 2010). This conception is for example illustrated by Duff and Logie 
(2001) who assumed independence between processing and storage within the verbal domain. However, a weaker version allows for some interference between processing and storage. For example, Baddeley and Logie (1999, p. 29) described the peripheral systems as "specialized for the processing and temporary maintenance of material within a particular domain”. Thus, processing should interfere with storage when both functions rely on the same domainspecific system. However, whatever the version retained, what the multiple-component view of working memory excludes is central interference by which activities involving two distinct domains (e.g., verbal and spatial or auditory and visual) would interfere with each other. Nonetheless, there is abundant evidence of between-domain interference between processing and storage, suggesting that the two functions rely on the same attentional resource.

Evidence for such a domain-general attentional mechanism in charge of both processing and storage was provided, for instance, by Stevanovski and Jolicoeur (2007) who reported disruption of visual working memory by a tone identification task as well as by Chen and Cowan (2009) who observed the disruption of verbal memory by a nonverbal choice RT task. In the same way, we observed that varying the cognitive load involved by a spatial task (judging the location of a black square appearing either in the upper or the lower part of the screen) has a direct impact on the maintenance of verbal information such as letters (Barrouillet et al., 2007; Portrat, Barrouillet, \& Camos, 2008). This was made even clearer in a more recent study in which verbal (remembering letters) and visuo-spatial storage (memory for locations) were combined with both verbal (semantic categorization) and visuo-spatial (visuo-spatial fit judgment) processing (Vergauwe et al., 2010). As Vergauwe et al. (2009) already established, increasing the cognitive load of concurrent visuo-spatial processing impaired visuo-spatial recall performance, but the same was true for increasing the cognitive load of concurrent verbal processing activities. Basically, the same pattern of results was 
observed for the verbal storage: increasing the cognitive load of both processing activities impaired verbal maintenance without any interaction.

In summary, the independence of processing and storage assumed by the multiplecomponent model with cognitive structures exclusively devoted either to storage or processing is no longer tenable. As the TBRS model predicted, there is a trade-off between processing and storage, any increase in the cognitive load involved by processing resulting in a correlative memory loss. However, the TBRS model goes further by predicting the function relating processing to storage.

\section{The mathematical function relating processing to storage}

The findings reported in the previous section provide evidence that concurrent processing has a disruptive effect on maintenance of information in working memory, and that this effect depends on temporal factors. Thus, the main function of working memory can be seen as achieving the optimal compromise between two highly adaptive but contradictory objectives, which are, on the one hand, maintaining active and ready for treatments as much information as possible and, on the other hand, processing the greatest amount of incoming relevant stimuli. The TBRS assumes that this compromise is achieved through time-sharing between the two functions. Both the transformation of working memory representations for processing purpose and their refreshing and maintenance for storage rely on executive processes that are constrained by a central bottleneck, thus resulting in the sequentiality between the two functions ${ }^{1}$. Theorizing the sequential functioning of working memory as resulting from a bottleneck constraining executive processes to fire one at a time allows us to relax the strong assumption of a focus of attention limited to only one element, as previous versions of the TBRS model assumed. Indeed, several empirical evidence suggest that this focus has a capacity of four instead of one element (Cowan, 1999, 2001; Cowan et al., 2005; Saults \& Cowan, 2007). What the time sharing observed between processing and storage in 
our previous studies suggests is the sequentiality of the two functions, but it does not necessarily result from a single-item attentional focus. Actually, this sequential functioning and the linear trend relating cognitive load to working memory span are not incompatible with alternative conceptions such as Cowan's embedded processes model and his assumption of a four-item focus of attention. In line with Cowan's proposal, it could be imagined that working memory representations can hold up to four items that can be embraced at a time within the focus of attention. This does not necessarily mean that the refreshing function could reactivate the entirety of the information held in these representations, especially when the time available for refreshing process becomes shorter and shorter as cognitive load increases. This partial and incomplete refreshing along with probabilistic retrieval mechanisms that do not lead to optimal recall even when memory traces are still in working memory can produce the observed linear function without assuming a one-item focus of attention constraining maintenance mechanisms to reactivate memory items one at a time.

Whatever the size of the focus of attention and the precise way by which the refreshing mechanism operates, the main point here is to keep the idea that while the central bottleneck is occupied for processing purposes, it is no longer available to refresh memory traces that suffer from a time-related decay. Thus, the working memory trade-off function relates the proportion of time occupied by processing activities, i.e. their cognitive load, to the amount of information that can be maintained active, usually assessed through working memory span measures. In this section, we establish that the TBRS model predicts the nature and the parameters of this function.

Working memory span tasks are especially appropriate to study the trade-off phenomenon by involving the two functions of processing and storage. If our hypothesis of a sequential functioning of working memory that alternates between processing and storage activities is correct, any temporal gap that can be freed during processing should be filled with 
maintenance activities, resulting in a perfect trade-off. This means that any variation in cognitive load $\delta C L$ should result in a correlative variation $\delta$ Span in the amount of information that can be maintained active. The relation between $\delta C L$ and $\delta$ Span should reflect the structure and functioning of the system in which this trade-off occurs.

A recent computational model of the TBRS permits one to specify the nature of this function (Oberauer \& Lewandowsky, in press). This computational model is aimed at simulating the time course of activation of the memoranda during working memory span tasks in which the presentation of each to-be-remembered item is followed by a series of processing episodes. When free time is available after a given processing episode, it is filled by refreshing. In a nutshell, the model assumes that each item to be memorized is represented by a single unit associated with a layer dedicated to the representation of its serial position. Thus, encoding an item consists in activating it in the item layer and associating it to its position by Hebbian learning. Each learning event is implemented as an exponential growth of connection strength over time towards an asymptote. At retrieval, the unit with the highest activation is selected, recalled, and then suppressed. In order to simulate the time-related decay hypothesized by the TBRS model, during every time step in the simulation, an exponential decay is applied to the whole weight matrix representing memory for a list. Refreshing is modeled as retrieval followed by a re-encoding of the retrieved item, with the only difference that this re-encoding is faster than the initial encoding and that, contrary to recall, the item refreshed is not suppressed from the list. In line with the sequential functioning of working memory assumed by the TBRS model, refreshing proceeds in a cumulative fashion, starting from the first list item and proceeding forward until the end of the list. When refreshing is interrupted by a processing episode or a new item, it is resumed starting from the first list item. Oberauer and Lewandowsky (in press) ran a simulation of a working memory span task in which they manipulated the cognitive load of processing by varying the duration of both 
processing episodes and free time, with 4 or 8 processing episodes after each memory item for lists varying in length from 1 to 9 . Recall performance was evaluated following the span procedure we adopted in most of our studies (e.g., Barrouillet et al., 2004). The simulation revealed a smooth and roughly linear decline of working memory spans with increasing cognitive load, suggesting that the function relating span to cognitive load is of the form:

$$
\text { Span }=a-b C L
$$

Actually, several empirical findings confirmed this linear relation between cognitive load and span in working memory tasks. For instance, using the reading digit span task already evoked, Barrouillet et al. (2004) observed that working memory span varied linearly with the pace at which the concurrent reading digit task was performed (Figure 1). Linear relations were also observed when investigating the visuo-spatial domain (Vergauwe et al., 2009), in working memory tasks involving between-domains combinations of storage and processing (e.g., verbal and visuo-spatial, Barrouillet et al., 2007; Vergauwe et al., 2010), as well as in children from age 7 onwards (Barrouillet, Gavens, Vergauwe, Gaillard, \& Camos, 2009).

We can go further by predicting the parameters of this linear function that can be directly derived from the main tenets of the TBRS. If we assume that working memory representations are transient in nature and suffer from a time-related decay when attention is switched away, prolonged periods without any refreshing activity should lead to the complete disappearance of these representations. Thus, in principle, a situation in which processing would continuously involve executive processes and occupy the central bottleneck for an extended period of time would result in a complete loss of working memory content. The parameters of the trade-off function should be as such as when $C L$ tends towards its maximum value 1 , working memory span tends towards 0 . As a consequence, equation (2) can be simplified as 


$$
\text { Span }=k(1-C L)
$$

where $k$ is a unique parameter reflecting both the slope and the intercept of the function. The value of this intercept corresponds to working memory span in a situation in which $C L=0$, all the time being available and devoted to maintenance activities. This situation should reflect what is observed in short-term memory tasks in which individuals concentrate all their capacities on the maintenance of a maximum number of items. Miller (1956) established that, in this situation, adults can maintain a limited number of $7 \pm 2$ chunks in working memory ${ }^{2}$. Thus, when $C L$ tends towards 0 , adults' working memory span should tend towards a value close to Miller's magical number. It is worth to note that the value $k$, the amount of information that can be maintained without any concurrent activity, varies as a function of the type of information to be maintained (letters, digits, or words) and age (Dempster, 1981), but also across individuals.

In summary, the TBRS model predicts that the amount of information that can be maintained active should linearly decrease with the increase of the cognitive load involved by concurrent processing, from a value close, in adults, to Miller's number for a null cognitive load to 0 for a cognitive load of 1 . This cognitive load corresponds to the proportion of time during which executive functions involved by this concurrent processing occupy the central bottleneck. In the next section, we provide empirical evidence supporting the trade-off function.

\section{An empirical test}

A proper test of the trade-off function would necessitate precise measures of cognitive load by evaluating the time during which a given activity effectively involves the central bottleneck, something difficult to do both for theoretical and methodological reasons because it is hardly possible to disentangle executive to non-executive processes within a given activity. However, apart from some experiments run in our laboratory within the TBRS 
theoretical framework (e.g., Barrouillet et al., 2007), we are not aware of a study that would have systematically investigated the effects on working memory spans of variations of cognitive load while carefully controlling for temporal parameters associated with the processing component of the tasks. To fill this gap, we performed a series of studies presented below with the aim of overcoming the difficulty of a precise evaluation of the time during which executive functions are involved by the processing component of working memory span tasks. Our strategy was to compare the duration of processing and the resulting recall performance on two working memory span tasks with processing components that differed only by the involvement of an executive function. For example, in one experiment, the tasks under comparison involved the maintenance of series of numbers, each number being followed by a Stroop task, the participants being asked to name the color of words. In one condition, the words displayed on screen were neutral words without any semantic relation with colors whereas, in the other, they were color words with $50 \%$ incongruent trials (e.g., the word "blue" colored in red). The prepotent response of reading the word displayed on screen was conceived of as creating a response conflict and the need to inhibit it to name the color, whereas the neutral words were used as a control condition involving a lower level of conflict. Thus, the difference in processing times between the two conditions could only be attributed to the extra involvement of the central bottleneck for inhibition, thus leading to a "pure" difference in cognitive load $\delta C L$. We reasoned that the resulting difference in recall $\delta$ Span could be related to $\delta C L$, providing us with a good approximation of the slope of the trade-off function $(\delta$ Span / $\delta C L)$.

Because there is no consensus about a finite and exhaustive list of what could be considered as executive functions, we adopted a comprehensive approach by exploring the effects on concurrent maintenance of two mechanisms classified as executive functions in the pivotal article of Miyake et al. (2000), namely the inhibition of prepotent responses and the 
updating of working memory content. Moreover, we summarize previous experiments investigating the effect on maintenance of the executive processes of response selection and directed retrieval from long-term memory, the results of which will be included with the new findings in a meta-analysis. All the studies presented below have the same rationale. Participants were presented with computer-paced working memory span tasks combining memory requirement with a concurrent processing task, each to-be-remembered item being followed by a series of stimuli to be processed. We varied the cognitive load involved by this intervening processing by manipulating either the stimuli or the task to be performed on them in order to induce an additional demand on executive control. The dependent variable was the maximum number of items that can be recalled (i.e., working memory span). In line with Equation (3), the relation between cognitive load and working memory spans should be linear with intercept values close to Miller's magical number $7 \pm 2$ and negative slopes values close to these intercepts.

\section{The inhibition of prepotent responses}

In a first series of experiments, variations in cognitive load were obtained by manipulating the need to inhibit prepotent responses in the processing component of timecontrolled working memory span tasks. For this purpose, we performed two experiments using a Stroop-like task paradigm. In the first experiment described above, participants had to name the color of either color words or neutral words. We verified in a pretest that the interference created by the incongruent trials resulted in longer response times in the colorword than in the neutral-word condition. Response times were measured from stimulus onset to the onset of the response detected by a vocal key and revealed an average extra-time of 51 ms per word in the color-word condition $(668 \mathrm{~ms})$ compared to the neutral-word condition (617 ms). The rationale of the second experiment was the same as the first except that participants no longer maintained numbers but series of monosyllabic words and evaluated 
the numerosity of small dice-like patterns of 1 to 4 Arabic digits or letters presented successively on screen. The digits condition created the possibility of incongruent trials (e.g., a display of three "2") in which the displayed digits trigger a prepotent response conflicting with the target response corresponding to the size of the dice-like pattern, something that could not occur with letters (see methods and results in Appendix). A pretest confirmed that incongruent trials induced a Stroop-like effect with longer response times for sets of digits (603 ms) than sets of letters (545 ms).

As we expected, the inhibition of prepotent responses involved in processing the incongruent trials of the Stroop-like conditions (color-words and digits) had a detrimental effect on concurrent maintenance. The color-word condition elicited significantly poorer recall performance than the neutral-word condition (mean spans of 2.93 and 3.18 respectively), and recall performance was significantly poorer in the Stroop-like condition involving patterns of digits than in the control condition involving letters (mean spans of 3.27 and 3.72 respectively). In order to assess the parameters of the predicted linear function, the cognitive load involved by the processing component of each of the four experimental conditions was approximated by calculating the ratio between the total processing time of the stimuli displayed in the interval between two successive items to be memorized and the total time available to process them (i.e., the duration of this interval). For this purpose, we used the response times observed in the pretests. The total processing time was evaluated by multiplying these response times by the number of stimuli to be processed after each memory item. The mean spans observed on each of the four conditions (ranging from 2.93 to 3.72) were regressed on these cognitive load values (ranging from .628 to .513), revealing a linear trend accounting for $98 \%$ of the observed variance in recall performance with a slope of -6.78 and an intercept of 7.16. Thus, this first study gave promising results with parameters of the linear relation within the expected range. 


\section{Working memory updating}

In a second series of experiments, variations in cognitive load were induced by manipulating the updating requirements of the processing component of the tasks. For the first experiment, we used a memory-updating task inspired from Oberauer (2002) who asked participants to keep track, in a self pace mode, of up to 6 simultaneous running counts related to different frames. The trials began with the presentation of an initial value (a digit) in each frame. Then, participants had to apply sequential arithmetic operations (e.g., +3 or -6$)$ appearing at random in the different frames, to update the corresponding value, and to go on the next step. After 9 updates, participants had to report the final value corresponding to each frame. We used a computer-paced version of this task in which participants had to perform only 2 running counts with simpler arithmetic operations (i.e., $+1,-1,+2$, and -2 ). For each new update, participants had to say aloud the answer of the current operation while maintaining the value that had not been updated. This task was inserted as processing component within a working memory span task in which participants had to maintain and recall series of letters. This updating condition was compared to a simple storage condition in which no updating was required. After the initial presentation of two frames with a digit in each frame, the successive arithmetic operations were replaced by a signal appearing in one of the two frames and participants had only to retrieve and utter the initial value presented in the corresponding frame without any modification of this value. The response times to each stimulus were recorded on-line using a voice key (see method in Appendix). As we expected, when compared with the simple storage task, the memory updating task led to longer processing times (mean response time of $994 \mathrm{~ms}$ and $493 \mathrm{~ms}$ respectively) and poorer recall performance (mean spans of 2.35 and 3.84 respectively).

The rationale of the second experiment was the same. Participants had to maintain series of numbers while performing an intervening N-back on series of letters. This task 
required participants to judge if the currently displayed letter was the same as to the one presented $\mathrm{N}$ trials before by pressing appropriate keys. Variations in cognitive load were obtained by manipulating the $\mathrm{N}$ value. In the updating condition, participants performed a traditional 2-back task on series of 8 letters presented after each number. This updating condition was compared with a 0 -back condition that did not require any updating. Participants were asked to indicate whether each letter corresponded to one of the first two letters of the series. Thus, both tasks were identical in their memory requirement with 2 letters to be maintained throughout the series to be processed, except that these letters had to be continuously updated in the 2-back condition whereas they remained unchanged for a given series in the 0-back condition. Accuracy and response times were registered (see method in Appendix). As it could be expected, the 2-back condition elicited longer reaction times than the 0-back condition (594 ms and $518 \mathrm{~ms}$ respectively), and lower spans (3.65 and 4.19 respectively).

The cognitive load in each of the four experimental conditions was evaluated in the same way as for the inhibition study as the ratio between the time needed to process the stimuli (i.e., the mean sum of the response times to the stimuli presented within the intervals between two successive memoranda) and the time allowed to process them (i.e., the duration of these intervals). These values ranged from .247 to .454 . For sake of comparison with the previous analyses, the working memory spans observed in the updating experiments were increased by 2 units to take into account the fact that, in each condition of both experiments, participants had to continuously maintain 2 items during the processing tasks on top of the tobe-recalled memory items. The resulting mean spans (ranging from 6.19 to 4.35 ) were regressed on these cognitive load values. The linear regression analysis revealed a high $\mathrm{R}^{2}$ value (.99), with an intercept within the expected range (8.18) and a slope close to this value (-8.48). In summary, the results concerning updating confirmed those observed in the 
inhibition studies. The additional involvement of these executive functions resulted in degraded memory performance with a linear relation between cognitive load and working memory spans. Both linear functions exhibited parameters in the range of the expected values.

\section{Response selection and retrieval}

In a previous study that aimed at investigating the critical role of temporal factors on the effect of processing on storage, Barrouillet et al. (2007, Exp. 3) studied the effect on maintenance of two tasks involving response selection and memory retrieval. These experiments are relevant for our purpose because response selection is known to compete with memory retrievals for the central bottleneck (Rohrer \& Pashler, 2003) and has accordingly been proposed to involve executive control (Hegarty, Shah, \& Miyake, 2000; Rowe, Toni, Josephs, Frackowiak, \& Passingham, 2000), whereas memory retrieval is considered by Baddeley (1996) as one of the functions of the central executive.

We used a computer-paced working memory span task in which participants were presented with series of letters to be remembered, each letter being followed by a series of digits displayed either in the upper or lower part of the screen. According to the condition they were assigned to, participants had to judge either the parity or the location on screen of these digits by pressing appropriate keys. The pace of these intervening activities and the resulting cognitive load was varied by manipulating the number of digits (either 4,6, or 8 ) presented in a constant interletter interval. The results revealed, as in the studies reported above, a strong effect of pace in both tasks with lower spans in the parity $(5.16,4.58$, and 3.69 for 4,6 , and 8 digits respectively) than in the location condition $(5.56,5.52$, and 4.60 respectively). This effect reflected the longer response times elicited by the parity task (a mean of $554 \mathrm{~ms}$ compared to $411 \mathrm{~ms}$ for the location task), which necessitates an additional retrieval from long-term memory compared to the location task that only involves response selection. In each of the 6 experimental conditions, the cognitive load involved by the 
processing component was approximated by dividing the mean total processing time by the total time allowed (i.e., the duration of the interletter intervals). When the mean span scores were regressed on this cognitive load, it appeared that the two slopes (- 7.82 and -7.68 for the parity and the location tasks respectively) were very close to each other and close to the corresponding intercepts (8.04 and 7.84 respectively), leading further support for the predicted equation.

\section{A meta-analysis}

The results of the inhibition and updating studies were included in a meta-analysis with the results of the experiments involving the parity and the location tasks from Barrouillet et al. (2007) that evaluated the impact of the functions of retrieval and response selection respectively. The main results of these different studies are summarized in Table 1. As our theory predicts, the intercepts of the trade-off functions range from 7.16 to 8.18 . This restricted range of intercept values corresponds to Miller's magical number, which is all the more remarkable since these experiments did not involve the same memory material (either letters, digits, or monosyllabic words). Moreover, some processing components involved a concurrent articulation, such as the experiments about inhibition, which is known to impair maintenance of verbal information. Accordingly, these experimental conditions resulted in a smaller intercept value than the others ( 7.16 compared to values near to 8$)$. The second prediction that slopes should be close to the intercept values is also supported by empirical evidence, the slope / intercept ratios ranging from 0.95 to 1.04 , all values very close to 1 . The mean observed intercept was 7.81 and the mean slope -7.69 , for a ratio of .985 . Finally, the meta-analysis regrouping the 14 experimental conditions reveals a linear function with an intercept of 8.13 and a slope of -8.33 (Figure 2), the linear regression accounting for $98 \%$ of the variance observed in the working memory spans. 
Thus, the variation in span resulting from the manipulation of the cognitive load induced by a variety of tasks and processes fitted the processing-storage equation predicted by the TBRS. Moreover, it should be noted that, in line with this model, the executive functions studied in this article have an effect on concurrent maintenance that does not mainly depend on their nature but on the time during which they occupy the central bottleneck and impede refreshing. This is a further evidence that time is a crucial factor in working memory functioning beyond and above the nature of its operations and of the representations processed.

\section{Some comments about the processing-storage equation}

Though the experimental data fitted the expected linear function, some prudence is needed in interpreting these results. First of all, it is worth noting that the trade-off function described here is not a working-memory-loss or a time-loss function. Indeed, the function only describes how the amount of information that can be maintained and recalled evolves with the temporal density of concurrent processing activities. It does not say anything about the speed of the time-related decay of working memory traces, because cognitive load refers to a time ratio and not to raw durations. Of course, the fact that recall performance depends almost entirely on the temporal aspects of the tasks strongly suggests that time plays a major role in working memory functioning and forgetting, but our data do not convey information about the function relating time to forgetting.

Second, the cognitive load values reported here are probably coarse estimates of the proportion of time during which the processing tasks really occupied the central bottleneck. Processing times measured from stimulus onset to response production probably encompass some cognitive steps that do not involve executive functions, such as the first steps of perceptual encoding or the realization of motor responses. The present experiments led to the predicted values probably because the slopes were calculated from experimental conditions 
that were designed to differ only on executive functions involvement. For example, it is quite certain that the difference in processing times between the Stroop-like and the control conditions in the inhibition experiments only resulted from the involvement of an additional inhibitory process. By contrast, processing times for more automatic activities, such as reading digits as Barrouillet et al. (2004) used, are probably poorer estimates of the involvement of central processes, even if these processes are involved in such simple activities (Barrouillet, Lépine, \& Camos, 2008).

Third, the trade-off function described here characterizes working memory functioning under some conditions. For example, the proportion of time corresponding to the cognitive load makes sense only for sufficiently extended periods of time. It seems highly improbable that, for a given cognitive load, very short and long periods of time would have the same effect on maintenance. It can not be expected that even a high cognitive load during very short periods of time (e.g., 1 second) would have a highly disruptive effect on maintenance, because most of the information previously activated can probably be retrieved after short interruptions. Finally, the entire trade-off function is extrapolated from cognitive load values ranging approximately from 0.25 to 0.65 . The other parts of the function, and more particularly those close to $C L=1$, are theoretical rather than empirical, although Oberauer et al. (2009) were able in their simulation to increase the cognitive load up to .90 and their results fitted the predicted linear trend. The null span value given by the trade-off function for a $C L$ of 1 confirms the fact that cognitive load is the main determinant of storage in working memory: A difference $\delta \mathrm{CL}$ of 0.1 results in a decrease in span corresponding to $\mathrm{k} x 0.1, \mathrm{k}$ being the maximum span with no concurrent activity (i.e., the simple span). Now, this is not to say that designing or performing a task that would continuously capture attention for a long period of time is possible, and that such a task would lead to a complete working memory loss. Actually, we never observed a complete memory loss, even in children, and we are not 
aware of any study reporting such a phenomenon. Even in the most demanding conditions of our computer-paced working memory span tasks, adults seem able to recall about 2 items (e.g., Barrouillet et al., 2004, Exp. 7) and 8-year-old children 1 item (Barrouillet et al., 2009). In line with our theory, what the equation of the trade-off function tells us is that, in the hypothetical case of a task occupying continuously the central executive during an extended period of time, the concurrent maintenance would become impossible and would result in a complete loss of working memory representations. Thus, exactly like some physical laws, the trade-off function and its extreme span values for CL tending towards 1 must be understood as a theoretical equation describing an ideal functioning that does not necessarily correspond to real physical or psychological states.

\section{A TBRS account of Baddeley \& Hitch (1974)}

As we previously noted, the seminal investigations of the relationships between processing and storage by Baddeley and Hitch (1974) led them to conclude to a relative independence between processing and storage. In contradiction with this conclusion, we have presented in this article several empirical evidence pointing towards a perfect time-based trade-off between the two functions, as the TBRS model predicted. However, a pending question concerns the capacity of our model to account for Baddeley and Hitch's (1974) findings and other results taken as evidence supporting the multi-component model and its fractionation between processing and storage. In this section, we show that these results contradict the hypothesis of a continuous resource-sharing, but are fully compatible with our hypothesis of a time-based resource sharing.

In most of Baddeley and Hitch (1974) studies, participants were presented with a reasoning task in which they had to judge the truth-value of a sentence purporting to describe the order of occurrence of two letters (e.g., $A$ is not preceded by $B-\mathrm{AB}$ ), this sentence remaining visible until participants pressed the "true" or "false" response key. The difficulty 
of the task was varied by using passive rather than active sentences and negative instead of affirmative propositions. This task was performed alone or while maintaining a concurrent preload of 1, 2 or 6 letters or digits. The results are well known. There was no reliable effect of memory load on accuracy and solution time regardless of whether the load was one or two items. With 6 items, memory load had a negligible effect on accuracy but slowed down reasoning considerably, an effect also observed when the processing was a task of language comprehension or free recall of unrelated words. A related unpublished study by Baddeley and Lewis reported in Baddeley (1986) involved the same reasoning task with a preload from 1 to 8 digits. Once more, memory load had virtually no effect on reasoning performance, but it appeared that the slowing down in reasoning times increased with the number of digits to be maintained (Figure 3). More interestingly, five days of practice did not remove the influence of concurrent load, and this effect of concurrent load did not interact with the difficulty of the reasoning task. The same function relating memory load to processing time was observed by Baddeley, Lewis, Eldridge, and Thomson (1984) with a sentence verification task.

Though these findings were taken as evidence for independence between processing and storage, they are actually compatible with our model in which maintenance activities and reasoning processes are underpinned by the same supply through a time-based sharing. In line with the hypothesis of a sequential functioning of working memory that alternates between processing and storage, any activity of refreshing memory traces would postpone concurrent processing steps for a duration commensurate with the amount of information to be maintained. Actually, as a time-based trade-off between processing and storage would predict, Baddeley (1986) observed that increasing the number of digits to be maintained resulted in a smooth increase in the time needed to perform the concurrent task. Interestingly, as shown in Figure 3, the relation between memory load and processing time was roughly linear, as the reverse function between cognitive load and working memory span is. Because the task takes 
only $2 \mathrm{~s}$, its postponement for refreshing purpose is probably not needed when few items have to be maintained, hence the total absence of effect for memory loads of one and two digits. The increase in processing time of about $720 \mathrm{~ms}$ for a load of 8 digits echoes the parameters of the computational simulation of the TBRS by Oberauer et al. (2009) who set at $80 \mathrm{~ms}$ the time needed to refresh a memory item.

Our model accounts also for the fact that this postponement has a small impact on performance in Baddeley and Hitch's task for two reasons. First, because information to be processed in the reasoning task is always available on screen, processing can be postponed without any damage for response accuracy. Second, our model assumes that there is no continuous sharing of attention, but a time-based sharing. As a consequence, when attention is turned to the processing task, all the capacities of the cognitive system are available and devoted to this processing, whatever the amount of information to be concurrently maintained. This can also explain that the effect of concurrent load did not interact with the difficulty of the reasoning task in Baddeley and Hitch (1974). Because the temporal effect of concurrent load on reasoning reflects a postponement consecutive to the activities of maintenance of the memoranda, this postponement depends only on the amount of information to be refreshed, but in any way on the difficulty of the postponed task. In the same way, as Baddeley and Hitch observed, practicing the task even during 5 days can not remove the need to reactivate memory traces and the ensuing postponement of the reasoning task.

Thus, instead of ruling out the idea that there is a common supply shared by processing and storage, Baddeley and Hitch's (1974) results were only at odds with the idea that processing and storage compete for the simultaneous occupation of a limited space, but they are compatible with a time sharing account, as the authors themselves noted in their discussion. 
Another and more recent refutation of a trade-off between processing and storage was alleged by Duff and Logie (2001). They assessed independently a word span and a sentence verification span (i.e., the maximum number of short sentences that participants could read and verify in $10 \mathrm{~s}$ ), and then had participants perform the two tasks in combination. The combined task followed the same logic as the Daneman and Carpenter's (1980) reading span task: participants performed the same task as in the sentence verification task but had to remember the last word of each sentence to be verified. The authors noted that, although the reduction in performance induced by the combined task was statistically robust, it was far from the dramatic decrease in performance that might have been expected if processing and storage were fuelled by a common pool of resource. As a consequence, they argued for a multiple resource model. As we have already argued elsewhere (Barrouillet et al., 2007), the facts reported by Duff and Logie (2001) are not at odds with a time-based resource sharing between processing and storage. Actually, both the word span and the verification span were about 4.75 when performed in isolation (i.e., a mean of $2.11 \mathrm{~s}$ per sentence). When the two tasks were performed in combination, the verification span dropped to 3.6 and the word span to 3 . Thus, the concurrent maintenance of words resulted in a slowing down of verification, with each sentence being now processed in a $2.78 \mathrm{~s}$. What Duff and Logie have demonstrated is that it takes an additional time of about $650 \mathrm{~ms}$ per sentence to concurrently store and maintain 3 words in a reading span task procedure. This result says nothing about the existence of multiple instead of a unique pool of resources and is perfectly in line with what our model would predict. The additional requirement of maintaining the last words of the sentences results in an overall increase in the time needed to process each of them.

In summary, the empirical evidences usually considered as supporting a fractionation between processing and storage are issued from experiments based on the same rationale: If processing and storage share a common resource, exhausting the storage capacity of working 
memory should have a dramatic impact on concurrent processing. However, this research strategy can only rule out models in which a unique resource is continuously shared between processing and storage, but not alternative views assuming the time-based sharing of a common supply. Actually, these studies reveal that memory load postpones processing exactly as the TBRS predicts.

\section{Alternative proposals and empirical evidence challenging the TBRS model}

Though our model was successful in predicting the mathematical function governing the trade-off between processing and storage, one of its main tenets, the hypothesis of forgetting in the short term through temporal decay, has been the object of recent evaluations and rebuttals. Naturally, these criticisms have been extended to the TBRS itself. In this section, we evaluate the findings on which these criticisms are based and show that there is no current evidence contradicting our theory.

\section{Evidence against temporal decay?}

In a recent series of studies, Lewandoswky and colleagues argued having demonstrated that there is no temporal decay of memory traces in the short-term (see Brown \& Lewandowsky, 2010; Lewandowsky, Oberauer, \& Brown, 2009, for reviews). For example, Lewandowsky, Duncan, and Brown (2004) investigated the effect of time on immediate serial recall of letters by asking participants to repeat a suppressor ("super") either one, two, or three times before the recall of each letter, thus increasing the delay between study and output. The results revealed no effect of the number of suppressors uttered, suggesting that memory traces do not suffer any temporal decay. These results were replicated and extended by Oberauer and Lewandowsky (2008). Nonetheless, they noticed that utterance of the suppressor blocks any verbal rehearsal, but allows the attentional refreshing postulated by the TBRS model to take place and counteract the effect of decay. This could explain why increasing the number of "super" had no effect on memory performance. In order to block the attentional mechanism 
of maintenance, Oberauer and Lewandowsky added a choice reaction task to the utterance of the suppressor "super", creating a condition referred to as AS + CRT (for articulatory suppression + choice reaction task). Participants were presented with either one or four stimuli that appeared successively on screen. In response to these stimuli (either \& or \%), participants had to say "super" aloud and press the left or the right key respectively. The results revealed no effect of the number of distractors to be processed (see Lewandowsky, Geiger, and Oberauer, 2008, for a replication). Lewandowsky et al. (2008) concluded that these findings provide a strong case against temporal decay: even when verbal rehearsal and attentional refreshing are impeded, increasing the delay between encoding and recall does not result in more forgetting as the temporal decay hypothesis would have predicted.

These is no doubt that these results constitute an impressive body of empirical evidence demonstrating that forgetting in the short term does not depend on the delay between encoding and recall. However, the TBRS model does not assume that forgetting depends on this delay, but on the proportion of time during which the central bottleneck is occupied by concurrent activities over this delay. Do these findings constitute compelling evidence against this latter view? We will set aside the tasks in which the delay between encoding and recall only involves the utterance of a varying number of "super". As Oberauer and Lewandowsky (2008) themselves noted, this articulatory suppression places very little demand on the attentional bottleneck and does not block the mechanism of attentional refreshing, the existence of which is attested by both behavioral (Camos, Lagner, \& Barrouillet, 2009; Hudjetz \& Oberauer, 2007) and neurological (Raye et al., 2007) evidence. As a consequence, the "super" experiments, including Lewandowsky et al. (2004), can not provide any evidence against temporal decay. We will thus concentrate on the tasks requiring both articulatory suppression and attentional demand. Because there seems to be a consensus about the existence of an attentional refreshing mechanism that can counteract the potential effects of 
decay, it is of crucial importance to control the possibility for participants to surreptitiously turn their attention towards the memoranda when processing distractors. However, in all the conditions of the experiments run by Lewandowsky and colleagues, the tasks were self paced, participants displaying themselves on screen the next stimulus by pressing some key. Thus, it can not be assumed, as Lewandowsky et al. (2008) did, that their choice task placed continuous demands on the attentional bottleneck, because participants were free to postpone the processing of the next distractor in any time all along the task ${ }^{3}$. Accordingly, the reaction times reported by Oberauer and Lewandowsky (2008) in their AS + CRT condition are far longer than choice reaction times observed in similar tasks (e.g., Barrouillet et al., 2007). Thus, it is highly improbable that the AS + CRT task created the intended continuous occupation of the attentional bottleneck that is needed to draw conclusions against temporal decay. Contrary to what Lewandowsky and colleagues have repeatedly claimed (Brown \& Lewandowsky, 2010; Lewandowsky et al., 2009), they did not provide compelling evidence against decay yet.

\section{Evidence against the TBRS model?}

The criticisms addressed by Lewandowsky and colleagues to the hypothesis of a temporal decay have been naturally extended to the TBRS model. Oberauer and Lewandowsky (2008) argued that the findings related to their AS + CRT condition posed a challenge to the TBRS model. Is this the case? It is worth noting that our model predicts that recall performance depends on the pace at which distractors are processed, which determines $C L$, and not on their number. Thus, at a constant pace, varying the number of distractors should not have any effect on recall, a prediction that was empirically verified (Barrouillet et al., 2004; Gavens \& Barrouillet, 2004). For example, in Barrouillet et al. (2004, Exp. 3), participants were presented with series of letters for further recall, each letter being followed by series of either 2 or 4 arithmetic operations at a rate of one operation every $2 \mathrm{~s}$. At this 
constant pace, we observed no effect of the number of operations on recall performance, though it took longer to solve 4 than 2 operations. It is only on the extreme case of an intervening task that would involve a maximal CL of 1 that increasing the delay between encoding and recall would result in poorer memory performance. However, we have seen that it cannot be reasonably argued that the AS + CRT task involved such a cognitive load in Oberauer and Lewandowsky (2008). As a consequence, the TBRS model is not challenged by these results because it does not predict poorer performance when four stimuli were to be processed instead of one.

In a more recent study, Lewandowsky, Geiger, Morrell, and Oberauer (2010) investigated the effects of the duration and type of to-be-articulated distractors in a complex span task design. This new series of experiments aimed at testing the predictions of their interference model of STM, named SOB (for Serial Order in a Box, Farrell \& Lewandowsky, 2002), which assumes that forgetting occurs through novelty encoding. Novel or dissimilar items would be more strongly encoded compared to what was already memorized, while the repeated encoding of the same item should cause minimal additional interference and disruption of encoding into STM. They tested these hypothesis using complex span tasks in which participants had to remember consonants followed by bursts of words to be read, with either one word, three different words, or the same word repeated three times. In line with SOB predictions, the results revealed that articulating once or three times the same word after each consonant had the same detrimental effect on recall compared with a condition without any distractor. By contrast, bursts of different words involved poorer recall performance than the repetition of the same word even when articulation times were equated.

Lewandowsky et al. (2010) argued that the absence of a distractor duration effect for the repetition condition constitute a challenge for the TBRS model because they assumed that participants articulating distractors continuously, their manipulation pushed cognitive load to 
the maximum level that can be achieved with speaking as a distractor activity. It is not difficult to see that these findings do not contradict the TBRS assumptions in any way. The effects related to the repetition of the same word would be annoying for the TBRS only if this activity continuously blocked the central bottleneck, impeding attentional refreshing. However, as Oberauer and Lewandowsky (2008) acknowledged, this articulatory suppression cannot block the mechanism of attentional refreshing. As a consequence, repeating the same word, even continuously, does not block the refreshing mechanism postulated by the TBRS model and cannot push the cognitive load to its maximal level. The TBRS model also accounts for the difference observed in recall between a mere repetition of the same word and reading three different words. While the level of articulatory suppression was similar between the two conditions, the attentional demand was higher for the latter which required reading three instead of one word. This confound between novelty and attentional demand undermines Lewandowsky et al. (2010) results. Actually, when novelty of the distractors and attentional demand of their processing are disentangled while temporal factors are carefully controlled, it appears that novelty per se has no effect in recall performance (Plancher \& Barrouillet, 2010). In summary, none of the findings reported by Lewandowsky and colleagues can be taken as compelling evidence against the TBRS model.

\section{General Discussion}

In this article, we have argued and provided empirical evidence in favor of an equation that relates the proportion of time devoted to processing, corresponding to the cognitive load induced by this processing, to the amount of information that can be maintained active in working memory. This function reveals that working memory spans for a given type of items can be understood as a function of the simple span for these items and the cognitive load induced by concurrent processing, thus unifying short-term and working memory in a common theoretical framework and their respective spans in a single mathematical function. 
This theoretical proposal and the related findings have a series of implications for our understanding of working memory structure and functioning. In this general discussion, we evaluate the capacity of the main theories of working memory to account for the mathematical relation described here between processing and storage. Three main hypotheses have so far been put forward. The first consists in assuming the independence of processing and storage, such as in Baddeley's (1986) theory or Duff and Logie's (2001) proposal. We have seen that this position is no longer tenable and can not account for the relation existing between processing and storage. The second hypothesis assumes a continuous resource sharing between processing and storage, while the third suggests a time-based sharing, as in the TBRS model. We will concentrate on these two latter hypotheses.

\section{Continuous resource-sharing between processing and storage}

This conception, which underlies most of the working memory span tasks, assumes that processing and storage are fueled by a unique pool of resources that are shared at any moment between the two functions. This conception has been very popular and endorsed, among others, by Daneman and Carpenter (1980), Case (1985), Turner and Engle (1989), Just and Carpenter (1992), or Anderson, Reder, and Lebière (1996).

Case's theory assumes that there is a limited total processing space in which some part is devoted to processing, the operating space, the remaining part, named the short-term storage space, being available for maintenance purpose. Thus, this theory predicts a trade-off between processing and storage, any increase in the demand of processing resulting in a correlative decrease in the resources left available for storage. Accordingly, Case, Kurland, and Goldberg (1982) provided evidence that counting spans vary with the efficiency of the processing component of the task. However, it remains unclear how this theory could predict the mathematical function studied here. The notions of resources and cognitive load remained too vague in Case's proposal to permit quantified predictions. 
Just and Carpenter (1992) also assumed that a same capacity, which can be thought of as the maximum amount of available activation, is shared between processing and storage. Their theory is essentially an activation model assuming that representations receive activation, and that those representations activated above threshold are part of working memory and become available for processing. Processing, understood in the theory as computation, consists in manipulations of activation by production rules that propagate activation from one element to another. The same production is assumed to fire repeatedly over successive cycles until the activation of its target element has reached the threshold. One of the key assumptions of the theory is that activation is a limited resource. If the total amount of activation available is less than the activation needed to perform a task, activation is deallocated from some old representations, resulting in a forgetting by displacement. In the same way, if several productions are required and if the amount of activation that they try to propagate exceeds the capacity, their attempts at propagation are scaled back, resulting in an increase in the number of cycles required to bring an element to threshold, then slowing down processing. At a first sight, such a theoretical framework is not incompatible with the processing-storage function. It could be assumed that, as the cognitive demand of the processing component increases, attention is deallocated from memory items that are lost. However, though this theory is compatible with the findings reported here as all the theories assuming a resource-sharing between processing and storage, it lacks the metric of cognitive demand needed to predict the parameters of the trade-off function. Without such a metric, the predictions issued from this theory, as well as from Case's model, remain qualitative. Moreover, Just and Carpenter (1992) assume that all enabled processes can execute simultaneously. This fits uneasily with the fact that working memory spans are a function of the duration of processing, which strongly suggests a sequentiality of the activities of processing and storage. 
More recently, Lovett, Daily, and Reder (2000; see also Lovett, Reder, \& Lebière, 1999) have proposed a source activation theory of working memory akin to Just and Carpenter's conceptions. Inspired from ACT-R architecture, this theory assumes that working memory is a subset of all declarative memory corresponding to those declarative chunks that receive activation from the current goal and the relevant production rules. These production rules, which are of the form condition-action, can only fire one at a time. According to Lovett et al. (2000), working memory is not limited by the total amount of available activation as in Just and Carpenter's theory, but by the amount of source activation, which is a type of attentional supply that produces activation and must be divided among the items in the focus of attention. When a given item does no longer receive activation through attention, its activation suffers from a time-related decay. It seems that this account of working memory (see also Anderson et al., 2004), with the assumptions of sequential processing and decaying memory traces, is the most able to account for the function relating processing to storage. As a matter of fact, the ACT-R model was one of our sources of inspiration in proposing the TBRS model. It could be imagined that, as soon as the processing episode can be interrupted, the goal of maintaining active the memoranda would activate some production rule allocating source activation to the decaying memory traces. A source of difficulty could reside in the possibility, in ACT-R, to activate several items simultaneously, whereas the first versions of the TBRS model assumed that memory traces are reactivated sequentially. This sequential refreshing was implemented in the simulation that produced the linear function (Oberauer \& Lewandowsky, in press), and it remains to verify if a parallel refreshing process would result in a linear function when varying cognitive load.

Among the theories that consider working memory as the activated part of long-term memory, the embedded-process model proposed by Cowan $(1999,2005)$ and Engle's controlled-attention theory (Engle, 2002; Engle et al., 1999; Engle \& Kane, 2004) are 
nowadays the most prominent. Both theories endorse a unitary view of working memory and assume a unique resource, attention, that fuels processing and storage. However, these models are not intended to give a detailed account of the relationships between processing and storage. Thus, they should potentially be able to account for the processing-storage function, but would need adequate extensions and additional assumptions.

\section{Time-based resource-sharing between processing and storage}

According to this last hypothesis, there is no continuous sharing of resource between the two functions but alternation, working memory switching from one function to the other. This is the hypothesis favored by the TBRS model. This task switching assumption has been recently endorsed by other models like Towse and Hitch (1995; Hitch, Towse, \& Hutton, 2001) and Saito and Miyake (2004).

Towse and Hitch (1995) assumed a task switching between processing and storage as well as a time-related decay of memory traces as soon as attention is switched away. However, this task switching differs from that assumed by the TBRS model because it would reflect the way the working memory span tasks are structured, disallowing a switching during the completion of the processing component. Rather, attention would be directed toward the processing activity and switched to storage only when memory items are presented. Thus, according to this theory, the amount of information that can be maintained would mainly depend on the raw duration of processing that determines the time during which memory traces decay. One of the consequences of this approach that departs from the TBRS model is to consider as superfluous the notion of cognitive load. Thus, Towse and Hitch's theory does not account for the processing-storage function. Incidentally, a series of studies comparing the predictions of Towse and Hitch's model with those issuing from our theory clearly indicated that the TBRS model provided a better account of the results (Barrouillet et al., 2004). 
Though Saito and Miyake (2004) recognize that attentional demand is an important element that must be added to Towse and Hitch's task-switching model to explain working memory span performance, their account of the relationships between processing and storage could fail to account for the results reported here. Indeed, Saito and Miyake (2004) assume that the forgetting mechanism during processing is not time-based, but rather representationbased, resulting from the degree of representational overlap.

As we have seen, the decay hypothesis has been the object of many criticisms (e.g., Lewandowsky et al., 2009; Unsworth, Heitz, \& Park, 2008), and as Saito and Miyake (2004) did, several theories of short-term memory (Brown, Neath, \& Chater, 2007; Nairne, 1990; Oberauer \& Lewandowsky, 2008) as well as working memory (Unsworth \& Engle, 2007) have claimed that forgetting occurs uniquely through representation-based interference. The main proposal of these approaches is clearly summarized by Unsworth and Engle (2007, p. 125) who state "many of the effects taken as evidence for decay-based models of working memory (e.g., Baddeley, 1986) can be handled by the cue-dependent retrieval frameworks". Of course, it can not be denied that interference is an important source of forgetting. We have elsewhere described how interference could occur within the TBRS model (Barrouillet et al., 2007; Camos et al., 2009; Camos, Mora, \& Oberauer, in press). Nonetheless, it will certainly be arduous to develop a purely interference-based model of forgetting fitting a trade-off function that so perfectly mimics the effect of time. One possibility would be to assume that the amount of interference created by the intervening activities is a direct function of their duration. However, interference-based theories of forgetting usually assume that interference results from the nature of the items involved by these intervening activities rather than from the time taken to process them (Oberauer \& Lewandowsky, 2008; Nairne, 1990). Another possibility would be to imagine that what matters is not the duration of occupation of the central bottleneck but the free time available to restore memory traces when attention is no 
longer occupied by processing episodes (Oberauer \& Kliegl, 2006; Lewandowsky \& Oberauer, 2009). According to this conception, memory traces would not suffer from a temporal decay during processing. The relationship between cognitive load and working memory spans would be due to the fact that lower values of cognitive load correspond to longer times during which attention is free and available to repair the damages created by interference. However, we have seen that activities that differ in nature have effects on storage commensurate with their duration (Figure 2). To account for this finding within a purely interference-based account of forgetting, it should be admitted that the amount of interference to be repaired does not depend on the nature of the stimuli to be processed, something at odds with the tenets of the interference theories.

In summary, several theories of working memory could accommodate the law relating processing to storage, provided changes and additional assumptions concerning the role of time in working memory functioning. Paradoxically, apart from the TBRS model, the models that appear closer to this benchmark do not assume a time sharing but a continuous resource sharing between processing and storage. This is probably due to the fact that the resourcesharing models often assume a temporal decay of memory traces (Lovett et al., 1999) or a memory loss related to the increasing demands of processing activities (Just \& Carpenter, 1992) that are the two phenomena that explain the nature of the time-related processingstorage function.

\section{Conclusion}

As we recalled above, Baddeley and Hitch's (1974) seminal enquiry aimed at investigating the difference between working and short-term memory: Was the short-term store described by Atkinson and Schiffrin (1968) a good candidate for the role of working memory? Their answer was negative, and they suggested that storage and processing involve distinct structures and mechanisms. There is no doubt that this conclusion, and the model that, 
accordingly, Baddeley and Hitch (1974) delineated, launched one of the most important and active research domains of cognitive psychology. However, the two functions that Baddeley (1986) segregated into different subsystems appear closely related to each other. As the TBRS model predicts, the interplay between processing and storage in working memory can be described by a general trade-off function that reflects the temporal constraints inherent to the sequential functioning of a unique system in charge of both working memory functions. The parameters of this trade-off function conform to well-known values with high psychological significance, such as Miller's magical number, permitting to establish not only a theoretical but a mathematical link between working memory and short-term memory. We can thus refine the answer given by Baddeley and Hitch (1974) to their initial question: short-term memory is the working memory when it does not "work", or more precisely when it works for maintenance purpose only. Rather being a subsystem of working memory, short-term memory could be one of its extreme states in the dynamic interplay between the two functions of processing and storage. 


\section{References}

Allen, R. J., Baddeley, A. D., \& Hitch, G. J. (2006). Is the binding of visual features in working memory resource-demanding ? Journal of Experimental Psychology : General, $135,298-313$

Allen, R. J., Hitch, G. J., \& Baddeley, A. D. (2009). Cross-modal binding and working memory. Visual Cognition, 17, 83-102.

Anderson, J. R., Bothell, D., Byrne, M. D., Douglass, S., Lebiere, C., \& Qin, Y. (2004). An integrated theory of the mind. Psychological Review, 111, 1036-1060.

Anderson, J. R., Reder, L. M., \& Lebiere, C. (1996). Working memory: Activation limitations on retrieval. Cognitive Psychology, 30, 221-256.

Atkinson, R. G., \& Shiffrin, R. M. (1968). Human memory : A proposed system and its control process. In K.W. Spence, \& J.T. Spence (Eds.). The Psychology of Learning and Motivation : Advances in Research and Theory, (Vol 2, pp. 89-195). New York : Academic Press.

Baddeley, A. D. (1986). Working memory. Oxford : Calendron Press.

Baddeley, A. D. (1996). Exploring the central executive. The Quarterly Journal of Experimental Psychology, 49, 5-28.

Baddeley, A. D. (2000). The episodic buffer : A new component of working memory? Trends in Cognitive Sciences, 4, 417-423.

Baddeley, A. D. (2001). Is Working Memory Still Working? American Psychologist, 56, 851864.

Baddeley, A. D., \& Hitch, G. (1974). Working memory. In G.A. Bower (Ed.), Recent advances in learning and motivation (Vol 8, pp. 647-667). New York : Academic Press. 
Baddeley, A. D., Lewis, V., Eldridge, M., \& Thomson, N. (1984). Attention and retrieval from long term memory. Journal of Experimental Psychology: General, 113, 518-540.

Baddeley, A. D., \& Logie, R. H. (1999). Working memory : The multiple component model. In A. Miyake \& P. Shah (Eds.), Models of working memory (pp. 28-61). New York : Cambridge University Press.

Barrouillet, P., Bernardin, S., \& Camos, V. (2004). Time constraints and resource-sharing in adults' working memory spans. Journal of Experimental Psychology : General, 133, 83-100.

Barrouillet, P., Bernardin, S., Portrat, S., Vergauwe, E., \& Camos, V. (2007). Time and cognitive load in working memory. Journal of Experimental Psychology : Learning, Memory, and Cognition, 33, 570-585.

Barrouillet, P., Gavens, N., Vergauwe, E., Gaillard, V., \& Camos, V. (2009). Working memory span development : A Time-Based Resource Sharing Model account. Developmental Psychology,45, 477-490.

Barrouillet, P., Lépine, R., \& Camos, V. (2008). Is the influence of working memory capacity on high level cognition mediated by complexity or resource-dependent elementary processes ? Psychonomic Bulletin \& Review, 15, 528-534.

Broadbent, D. E. (1975). The magic number seven after fifteen years. In A. Kennedy \& A. Wilkes (Eds.), Studies in long-term memory (pp. 3-18), New York : Willey.

Brown, G. D. A., \& Lewandowsky, S. (2010). Forgetting in memory models: Arguments against trace decay and consolidation failure. In S. Della Sala (Ed.), Forgetting (pp. 49-75). Hove, UK: Psychology Press.

Brown, G. D. A., Neath, I., \& Chater, N. (2007). A temporal ratio model of memory. Psychological Review, 114, 539-576. 
Camos, V., Lagner, P., \& Barrouillet, P., (2009). Two maintenance mechanisms of verbal information in working Memory. Journal of Memory and Language, 61, 457-469.

Case, R. (1985). Intellectual development: Birth to adulthood. New York: Academic Press.

Case, R., Kurland, M., \& Goldberg, J. (1982). Operational efficiency and the growth of shortterm memory. Journal of Experimental Child Psychology, 33, 386-404.

Chen, Z., \& Cowan, N. (2009). How verbal memory loads consume attention. Memory \& Cognition, 37, 829-836.

Cohen, J. D., Perlstein, W. M., Brauer, T. S., Nystrom, L. E., Noll, D. C., Jonides, J., \& Smith, E. E. (1997). Temporal dynamics of brain activation during a working memory task. Nature, 386, 604-608.

Content, A., Mousty, P., Radeau, M. (1990). Brulex - A computerized lexical data-base for the French-language. Année Psychologique, 90, 551-566.

Cowan, N. (1999). An embedded-process model of working memory. In A. Miyake, \& P. Shah (Eds.), Models of working memory : Mechanisms of active maintenance and executive control (pp. 62-101). Cambridge : Cambridge University Press.

Cowan, N. (2001). The magical number 4 in short-term memory: A reconsideration of mental storage capacity. Behavioral and Brain Sciences, 24, 87-185

Cowan, N. (2005). Working memory capacity. Hove, UK : Psychology Press.

Cowan, N. (2010). The magical mystery four: How is working memory capacity limited, and why? Current Directions in Psychological Science, 19, 51-57.

Cowan, N., Elliott, E. M., Saults, J. S., Morey, C. C., Mattox, S., Hismjatullina, A., \& Conway, A. R. A. (2005). On the capacity of attention: Its estimation and its role in working memory and cognitive aptitudes. Cognitive Psychology, 51, 42-100. 
Cowan, N., Saults, J. S., \& Elliott, E. M. (2002). The search for what is fundamental in the development of working memory. Advances in Child Development and Behaviour, 29, $1-49$.

Daneman, M., \& Carpenter, P. A. (1980). Individual differences in working memory and reading. Journal of Verbal Learning and Verbal Behavior, 19, 450-466.

Dempster, F. N. (1981). Memory span: Sources of individual and developmental differences. Psychological Bulletin, 89, 63-100.

Duff, S. C., \& Logie, R. H. (2001). Processing and storage in working memory span. The Quarterly Journal of Experimental Psychology, 54, 31-48.

Engle, R. W. (2002). Working memory capacity as executive attention. Current Directions in Psychological Science, 11, 19-23.

Engle, R. W., \& Kane, M. J. (2004). Executive attention, working memory capacity, and a two-factor theory of cognitive control. In B. Ross (Ed.), The Psychology of Learning and Motivation (Vol. 44, pp. 145-199). NY : Elsevier.

Engle, R. W., Kane, M. J., \& Tuholski, S. W. (1999). Individual differences in working memory capacity and what they tell us about controlled attention, general fluid intelligence, and functions of prefrontal cortex. In A. Miyake, \& P. Shah (Eds.), Models of working memory : Mechanisms of active maintenance and executive control (pp. 102134). Cambridge : Cambridge University Press.

Ericsson, K. A., \& Kintsch, W. (1995). Long-term working-memory. Psychological Review, $102,211-245$

Farrell, S., \& Lewandowsky, S. (2002). An endogenous distributed model of ordering in serial recall. Psychonomic Bulletin \& Review, 9, 59-79. 
Gavens, N., \& Barrouillet, P. (2004). Delays of retention, processing efficiency, and attentional resources in working memory span development. Journal of Memory and Language, 51, 644-657.

Hegarty, M., Shah, P., \& Miyake, A. (2000). Constraints on using the dual-task methodology to specify the degree of central executive involvement in cognitive tasks. Memory \& Cognition, 28, 376-385.

Hitch, G., Towse, J. N., \& Hutton, U. (2001). What limits children's working memory span? Theoretical accounts and applications for scholastic development. Journal of Experimental Psychology: General, 130(2), 184-198.

Hudjetz, A., \& Oberauer, K. (2007). The effects of processing time and processing rate on forgetting in working memory: Testing four models of the complex span paradigm. Memory \& Cognition, 33, 1675-1684.

Just, M. A., \& Carpenter, P. A. (1992). A capacity theory of comprehension : Individual differences in working memory. Psychological Review, 99, 122-149.

Klauer, K. C., \& Zhao, Z. M. (2004). Double dissociations in visual and spatial short-term memory. Journal of Experimental Psychology-General, 133, 355-381.

Lépine, R., Bernardin, S., \& Barrouillet, P. (2005). Attention switching and working memory spans. European Journal of Cognitive Psychology, 17, 329-345.

Lewandowsky, S., Duncan , M., \& Brown, G. D. A. (2004). Time does not cause forgetting in short-tterm serial recall. Psychonomic Bulletin \& Review, 11, 771-790.

Lewandowsky, S., Geiger, S. M., Morrell, D. B., \& Oberauer, K. (2010). Turning simple span into complex span: Time for decay or interference from distractors? Journal of Experimental Psychology: Learning, Memory, and Cognition, 36, 958-978.

Lewandowsky, S., Geiger, S. M., \& Oberauer, K. (2008). Interference-based forgetting in verbal short-term memory. Journal of Memory and Language, 59, 200-222. 
Lewandowsky, S., \& Oberauer, K. (2009). No Evidence for Temporal Decay in Working Memory. Journal of Experimental Psychology: Learning, Memory \& Cognition, 35, $1545-1551$.

Lewandowsky, S., Oberauer, K., \& Brown, G. D. A. (2009). No temporal decay in verbal short-term memory. Trends in Cognitive Sciences. 13, 120-126.

Logie, R. H. (1995). Visuo-spatial working memory. Hillsdale, NJ : Lawrence Erlbaum.

Logie, R. H., \& Duff, S. C. (1996). Processing and storage in working memory : Multiple components? Poster presented at the annual meeting of Psychonomics Society, Chicago, IL.

Lovett, M. C., Daily, L. Z., \& Reder, L. M. (2000). A source activation theory of working memory: cross-task prediction of performance in ACT-R. Cognitive Systems Research, $1,99-118$.

Lovett, M. C., Reder, L. M., \& Lebière, C. (1999). Modeling working memory in a unified architecture : An ACT-R perspective. In, A. Miyake, \& P. Shah (Eds.), Models of working memory : Mechanisms of active maintenance and executive control (pp. 135182). Cambridge : Cambridge University Press.

Miller, G. A. (1956). The magical number seven plus or minor two : some limits on our capacity for processing information. Psychological Review, 63, 81-97.

Miyake, A., Friedman, N. P., Emerson, M. J., Witzki, A. H., Howerter, A., \& Wager, T. (2000). The unity and diversity of executive functions and their contributions to complex "frontal lobe" tasks : A latent variable analysis. Cognitive Psychology, 41, 49100.

Nairne, J. S. (1990). A feature model of immediate memory. Memory \& Cognition, 18, 251269. 
Oberauer, K. (2002). Access to information in working memory : Exploring the focus of attention. Journal of Experimental Psychology : Learning, Memory, and Cognition, 28, 411-421.

Oberauer, K. (2005). Control of the contents of working memory-A comparison of two paradigms and two age groups. Journal of Experimental Psychology : Learning, Memory, and Cognition, 31, 714-728.

Oberauer, K., \& Kliegl, R. (2006). A formal model of capacity limits in working memory. Journal of Memory and Language, 55, 601-626.

Oberauer, K., \& Lewandowsky, S. (2008). Forgetting in immediate serial recall : decay, temporal distinctiveness, or interference ? Psychological Review, 115, 544-576.

Oberauer, K., \& Lewandowsky, S. (in press). Modeling working memory: A computational implementation of the Time-Based Resource-Sharing theory. Psychonomic Bulletin \& Review.

Pashler, H. (1998). The psychology of attention. Cambridge, MA : MIT Press.

Plancher, G., \& Barrouillet, P. (2010, September). Does novelty encoding cause forgetting in working memory? Poster presented at the Fifth European Working Memory Symposium (EWOMS), Civita Castellana.

Portrat, S., Barrouillet, P., \& Camos, V. (2008). Time-related decay or interference-based forgetting in working memory? Journal of Experimental Psychology : Learning, Memory, and Cognition, 34, 1561-1564.

Raye, C. L., Johnson, M. K., Mitchell, K. J., Greene, E. J., \& Johnson, M. R. (2007). Refreshing: A minimal executive function. Cortex, 43, 135-145.

Repovs, G., \& Baddeley, A. D. (2006). The multi-component model of working memory : Explorations in experimental cognitive psychology. Neuroscience, 139, 5-21. 
Rohrer, D., \& Pashler, H. (2003). Concurrent task effects on memory retrieval. Psychonomic Bulletin and Review, 10, 96-103.

Rowe, J. B., Toni, I., Josephs, O., Frackowiak, R. S. J., \& Passingham, R. E. (2000). The prefrontal cortex : Response selection or maintenance within working memory? Science, $288,1656-1660$.

Saito, S., \& Miyake, A. (2004). On the nature of forgetting and the processing-storage relationship in reading span performance. Journal of memory and Language, 50, 425443.

Saults, J. S., \& Cowan, N. (2007). A central capacity limit to the simultaneous storage of visual and auditory arrays in working memory. Journal of Experimental Psychology: General, 136, 663-684

Stevanovski, B., \& Jolicoeur, P. (2007). Visual short-term memory: Central capacity limitations in short-term consolidation. Visual Cognition, 15, 532-563.

Towse, J. N., \& Hitch, G. J. (1995). Is there a relationship between task demand and storage space in tests of working memory capacity? The Quarterly Journal of Experimental Psychology, 48A, 108-124.

Turner, M. L., \& Engle, R. W. (1989). Is working memory capacity task dependant? Journal of Memory and Language, 28, 127-154.

Unsworth, N., \& Engle, R.W. (2007). The nature of individual differences in working memory capacity : Active maintenance in primary memory and controlled search from secondary memory. Psychological Review, 114, 104-132.

Unsworth, N., Heitz, R. P., \& Parks, N. A. (2008). The importance of temporal distinctiveness for forgetting over the short-term. Psychological Science, 19, 1078-1081. 
Vergauwe, E., Barrouillet, P., \& Camos, V. (2009). Visual and spatial working memory are not that dissociated after all : A time-based resource-sharing account. Journal of Experimental Psychology: Learning, Memory, and Cognition, 35, 1012-1028.

Vergauwe, E., Barrouillet, P., \& Camos, V. (2010). Do mental processes share a domaingeneral resource? Psychological Science, 21, 384-390. 


\section{Appendix}

\section{Method and results of the experiments reported in the section "An empirical test"}

All the experiments contrasted two conditions varying in the cognitive load involved by the processing component of the working memory span task. Participants were presented with series of to-be-remembered items of various lengths with 3 series of each length in both experimental conditions. Across participants, these series were counterbalanced between conditions. At the end of the series, the word "recall" was displayed on screen and participants were asked to write down the remembered items in correct order by filling out frames containing the appropriate number of boxes. They had to fill out the boxes serially from left to right and to leave a blank if they did not remember the item at a given place. Recall performance was assessed by computing span scores as in Barrouillet et al. (2007). Each correctly recalled series of to-be-remembered items counted as one third; the total number of thirds was added to 2 (the shorter series being of length 3 ) to provide a span score. For example, in a given experimental condition, the correct recall of the 3 series of 3 numbers, of 1 series of 4 numbers and 1 series of 5 numbers resulted in a span of $2+(3+1+$ 1) $x 1 / 3=3.67$. This span score reflects the maximum number of items that can be held in working memory while concurrently processing information. Each experiment involved a different group of undergraduate students at the Université de Bourgogne who received partial course credit for participating.

\section{Stroop-Color Task}

Twenty participants (mean age $=21.1$ years, $S D=1.3,18$ females) had to memorize series of 3 to 6 monosyllabic numbers from 1 to 16 in which repetitions were avoided. Depending on the experimental condition (Stroop-like or control), each number was followed by series of either 8 color words or neutral words successively displayed on screen, the color 
of which had to be named. The color words "bleu" (blue), "rouge" (red), "vert" (green), and “jaune" (yellow) appeared on screen either in blue, red, green, and yellow with $50 \%$ congruent trials. The neutral-words were adjectives, with the same length and the same frequency as the color words, but without any semantic relation with colors: "doux" (soft), "connu" (known), "rare" (rare), "utile" (useful). The 8 items of each series appeared in a fixed random order with the restriction that two consecutive items do not appear in the same color to avoid two identical successive responses.

Each trial began by a ready signal (an asterisk) for $750 \mathrm{~ms}$. After a delay of $500 \mathrm{~ms}$, the first number appeared for $1500 \mathrm{~ms}$ and was followed by a post-number delay of $500 \mathrm{~ms}$. Then, 8 words were successively displayed on screen for $667 \mathrm{~ms}$ with a post-word delay of $333 \mathrm{~ms}$, resulting in an inter-number interval of $8500 \mathrm{~ms}(500 \mathrm{~ms}+8$ [667 ms $+333 \mathrm{~ms}])$. After the last of the 8 post-word delays, the second number appeared, followed by another series of 8 words and so on until the end of the series. At this point, the word "recall" appeared on screen. The 24 series were presented following two random orders, participants being informed of the length of the forthcoming series (e. g., "3 items to memorize"). The experimental session was preceded by a training phase in which participants named the color of 32 color-words and 32 neutral-words, and then practiced one trial of the working memory task in both conditions. All participants reached a high rate of correct responses in both conditions of the color naming task with, on average, $99 \%$ and $97 \%$ of correct responses for neutral-words and color-words respectively. Recall performance was poorer in the color-word than in the neutral-word conditions (mean spans of 2.93, $S D=.74$, and 3.18, $S D=.82$, respectively), $t(19)=2.12, p<.05$.

Sroop-digit task

Twenty participants (mean age $=19.7$ years, $S D=1.5,13$ females) were presented with a complex span task that had exactly the same structure as the previous experiment, except 
that the memory items were series of 3 to 6 words and the items to be processed were sets of 1 to 4 digits $(1,2,3$, or 4$)$ or letters $(\mathrm{P}, \mathrm{M}, \mathrm{S}$, or $\mathrm{R})$ displayed on screen in canonical dice-like patterns, resulting respectively in Stroop-like and control conditions. Words were three-letter monosyllabic masculine nouns selected from the French Brulex database (Content, Mousty, \& Radeau, 1990). The number of items to be processed after each word, the proportion of incongruent trials in the Stroop-like condition, the time course of the different stimuli presented on screen, as well as the structure of the training phase were the same as in the previous experiment. All participants reached a high rate of correct responses in both conditions of the enumeration task with, on average, $100 \%$ for letters and $98 \%$ for digits. Recall performance was poorer in digit than in the letter condition (mean spans of $3.27, S D=$ .81 , and 3.72, $S D=.82$, respectively), $t(19)=2.80, p<.05$.

\section{Memory updating vs. simple storage}

Twenty-four participants (mean age $=19.8$ years, $\mathrm{SD}=1.5,23$ females) had to read aloud and memorize series of 3 to 8 consonants. There were three series of each length in each experimental condition (memory updating and simple storage) resulting in $18 \times 2=36$ series presented in random order. Before each series of letters, a signal displayed on screen for $1500 \mathrm{~ms}$ indicated the task to be performed (the letters "opé" for the memory updating task and a star for the simple storage task). Each letter was then presented during $1500 \mathrm{~ms}$ and followed, after a delay of $500 \mathrm{~ms}$, by two $5 \mathrm{~cm}$ x $5 \mathrm{~cm}$ square frames horizontally displayed on screen with a $7.5 \mathrm{~cm}$ interval. The stimuli appearing in these frames were as follows. First, the two frames were filled with a different digit randomly selected from 1 to 9 that remained on screen for $1 \mathrm{~s}$. After a delay of $500 \mathrm{~ms}, 8$ stimuli were successively displayed at random either in the left or the right frame for $1 \mathrm{~s}$ each and followed by a $500 \mathrm{~ms}$ delay. In the simple storage condition, these stimuli were stars, whereas in the memory updating condition, they 
were simple operands (either $-2,-1,+1$, or +2 ). To facilitate discrimination, the stimuli were always blue in the left frame and red in the right frame.

For the simple storage task, participants were asked to say aloud the digit that had been displayed in the same frame as the currently displayed star. For the memory updating task, they were asked to update the current value of the respective frame according to the currently displayed operation by saying aloud the intermediary result (the series of operations were constructed to avoid results lower than 1 or higher than 9). In both tasks, the experimenter checked accuracy on line while response times were registered using a voice key. Before performing the task, participants practiced the memory updating and the simple storage tasks with 9 series of 8 stimuli, and the working memory span tasks in both condition.

The on-line registration of responses times during processing induced a lot of technical failures, but $81 \%$ of response times were successfully recorded by the voice key. Five participants whose more than one third of the response times were lost were discarded from the analyses that were run on the 19 remaining participants. Despite its intrinsic difficulty and the additional burden of the memory load, the memory updating task elicited a high rate of correct responses $(79 \%)$ whereas performance on the simple storage task was quite perfect with $98 \%$ of correct responses. The memory updating task took longer than the simple storage task (mean response time of $994 \mathrm{~ms}$ and $493 \mathrm{~ms}$ respectively), $t(18)=39.38, p<.001$ and involved lower recall performance (mean spans of 2.35, SD =.46, and 3.84, $S D=1.17$, respectively), $t(18)=7.53, p<.001$.

\section{The N-back task}

Twenty-nine participants (mean age $=19.2$ years, $\mathrm{SD}=1.9,23$ females) performed two working memory span tasks in which the processing component was an $\mathrm{N}$-back task, the $\mathrm{N}$ value being either 0 or 2 . Half of the participants performed the 0 -back task first and the other half the 2-back task. In each task, they had to read aloud and memorize series of 3 to 7 
numbers from 1 to 16 . In both tasks, each number was followed by a series of 8 successive letters (consonants only). In the 2-back task, participants had to judge if the currently presented letter was the same as the one presented 2 trials before, whereas in the 0-back task, they had to judge if it was the same as one of the two first letters of the series (the targets). Thus, both tasks were identical in their memory requirement with 2 letters to be maintained throughout the series to be processed, except that these letters had to be continuously updated in the 2-back condition whereas they remained unchanged for a given series in the 0-back condition. In both conditions, participants were instructed to not respond on the two first letters that were easily distinguishable by their blue color (all the other letters were black). In both conditions and in each series, among the 6 following black letters, 2 elicited a positive and 4 a negative response, one of which being a lure. Lures were letters requiring a negative response but eliciting positive response based on familiarity feeling (Cohen et al., 1997; Oberauer, 2005). In the 2-back condition, lures were letters presented 3 or 1 trial before (and not 2). In the 0-back task, lures were either letters already presented in the current sequence, but not as targets, or letters that were targets, but in the previous series.

Irrespective of the task, each trial began by a fixation point that was displayed for 750 ms and followed, after a $500 \mathrm{~ms}$ delay, by the first to-be-remembered number appearing into a centered frame $(7.5 \mathrm{~cm} \times 7.5 \mathrm{~cm})$ for $1500 \mathrm{~ms}$. After a $500 \mathrm{~ms}$ post-number delay, the 8 letters were successively displayed for $1000 \mathrm{~ms}$ and followed by a $500 \mathrm{~ms}$ post-letter delay. Participants gave their response by pressing one of two identified keys (positive response on the right side). After the last of the 8 post-letter delays, the second number appeared, followed by 2 other targets and 6 other letters and so on up to the end of the series where the word "recall" appeared. Before the experimental sessions, participants familiarized with the forthcoming task on 15 sequences of 2 targets and 6 stimuli to process (with beeps for mistakes and a $80 \%$ correct response criterion) and practiced with the working memory task 
per se. Five participants who did not reach an average of $80 \%$ correct responses in the Nback tasks were discarded from the analyses that were run on the 24 remaining participants, half of them having performed the 0-back task first, and the other half the 2-back task first. Not surprisingly, the 2-back task was more difficult than the 0 -back task (88\% and $93 \%$ of correct responses respectively), $t(23)=7.13, p<.001$, and involved longer reaction times (594 $\mathrm{ms}$ and $518 \mathrm{~ms}$ respectively), $t(23)=6.75, p<.001$, and lower spans $(3.65, S D=1.13$, and 4.19, $S D=1.14$, respectively), $t(23)=18.60, p<.001$. 


\section{Author note}

Parts of this work have been presented in Sophie Portrat's doctoral dissertation, at the joint Psychonomic/EPS meeting (Edinburg, July 2007) as well at the fourth European Working Memory Symposium (EWOMS, Bristol, September 2008). This research was supported by grants from the Swiss National Science Foundation ( $\left.{ }^{\circ} 100014-122626\right)$, ANR (05-BLAN-0346-01) and the Région Bourgogne (FABER). The authors would like to thank Frederik DeBoeck for his assistance in collecting data for experiments on updating. Sophie Portrat is now at Université Pierre Mendès France, Grenoble, France, and Valérie Camos is at the University of Fribourg, Switzerland. Correspondence concerning this article should be sent to Pierre Barrouillet, FPSE Université de Genève, 40 bd du Pont d'Arve, 1211 Genève 4, Switzerland,E-mail: Pierrre.Barrouillet@unige.ch. 


\section{Footnotes}

1. The TBRS model assumes that memory traces are refreshed through a process of covert retrieval described by Cowan, Saults, and Elliott (2002), and identified by Raye, Johnson, Mitchell, Greene, and Johnson (2007) as a minimal executive function, distinct from articulatory rehearsal, and neurally located in the left dorsolateral prefrontal cortex.

2. Of course, further studies have suggested that the capacity of working memory should be scaled down from 7 to 4 (Broadbent, 1975; Cowan, 2001, 2010). However, these later estimates refer to the "pure" capacity of working memory when mechanisms of maintenance are blocked. What we are referring to here is the maximum number of verbal items that people can actually maintain in the short term when no other activity interferes with maintenance, which corresponds to Miller's magical number.

3. It could be argued that in our computer-paced span tasks, participants are also free to postpone processing for refreshing memory traces. However, in computer-paced span tasks, any postponement results in a reduction of the time available to perform the following item, the appearance of which does not depend on participant's activities. By contrast, postponing a processing episode in Lewandowsky et al.'s procedure has no impact on the time available to process the following stimuli. 
$\underline{\text { Table } 1}$

Proportion of explained variance $\left(\mathrm{R}^{2}\right)$, slope, intercept and slope / intercept ratio resulting from the regressions of span scores on cognitive load of the tasks involving executive functions of retrieval, response selection, inhibition and updating. The data concerning retrieval and response selection are from Barrouillet et al. (2007, Exp. 3) and refer respectively to the parity and location judgment tasks used in this study.

\begin{tabular}{lccccc}
\hline & \multicolumn{5}{c}{ Executive function } \\
\cline { 2 - 5 } & Retrieval & $\begin{array}{l}\text { Response } \\
\text { Selection }\end{array}$ & Inhibition & Updating & $\begin{array}{c}\text { Meta- } \\
\text { analysis }\end{array}$ \\
\hline $\mathrm{R}^{2}$ & .93 & .86 & .98 & .99 & .98 \\
Slope & -7.82 & -7.68 & -6.78 & -8.48 & -8.33 \\
Intercept & 8.04 & 7.84 & 7.16 & 8.18 & 8.13 \\
Ratio & .97 & .98 & .95 & 1.04 & 1.03 \\
\hline
\end{tabular}




\section{Figure caption}

Figure 1: Mean spans as a function of the number-of-digits-to-time ratio. Adapted from

“Time constraints and resource-sharing in adults' working memory spans," by P. Barrouillet, S. Bernardin, and V. Camos, 2004, Journal of Experimental Psychology: General, 133, p. 94. Copyright 2004 by the American Psychological Association.

Figure 2: The processing-storage trade-off function of working memory. Mean span as a function of the cognitive load involved by four different types of tasks. Data concerning response selection and retrieval from "Time and cognitive load in working memory", by P. Barrouillet, S. Bernardin, S. Portrat, E. Vergauwe, V. Camos, 2007, Journal of Experimental Psychology: Learning, Memory \& Cognition, 33, p. 577. Copyright 2007 by the American Psychological Association. The three values for response selection and retrieval are from Exp. 3 in Barrouillet et al. (2007) and correspond to the three conditions (i.e., 4, 6, and 8 stimuli) of the location and the parity judgment tasks respectively.

Figure 3: Response times and error rates in a reasoning task as a function of the concurrent digit load in Baddeley and Lewis’ study reported in Baddeley (1986). Adapted from, Working memory, Baddeley (1986), Oxford University Press. 


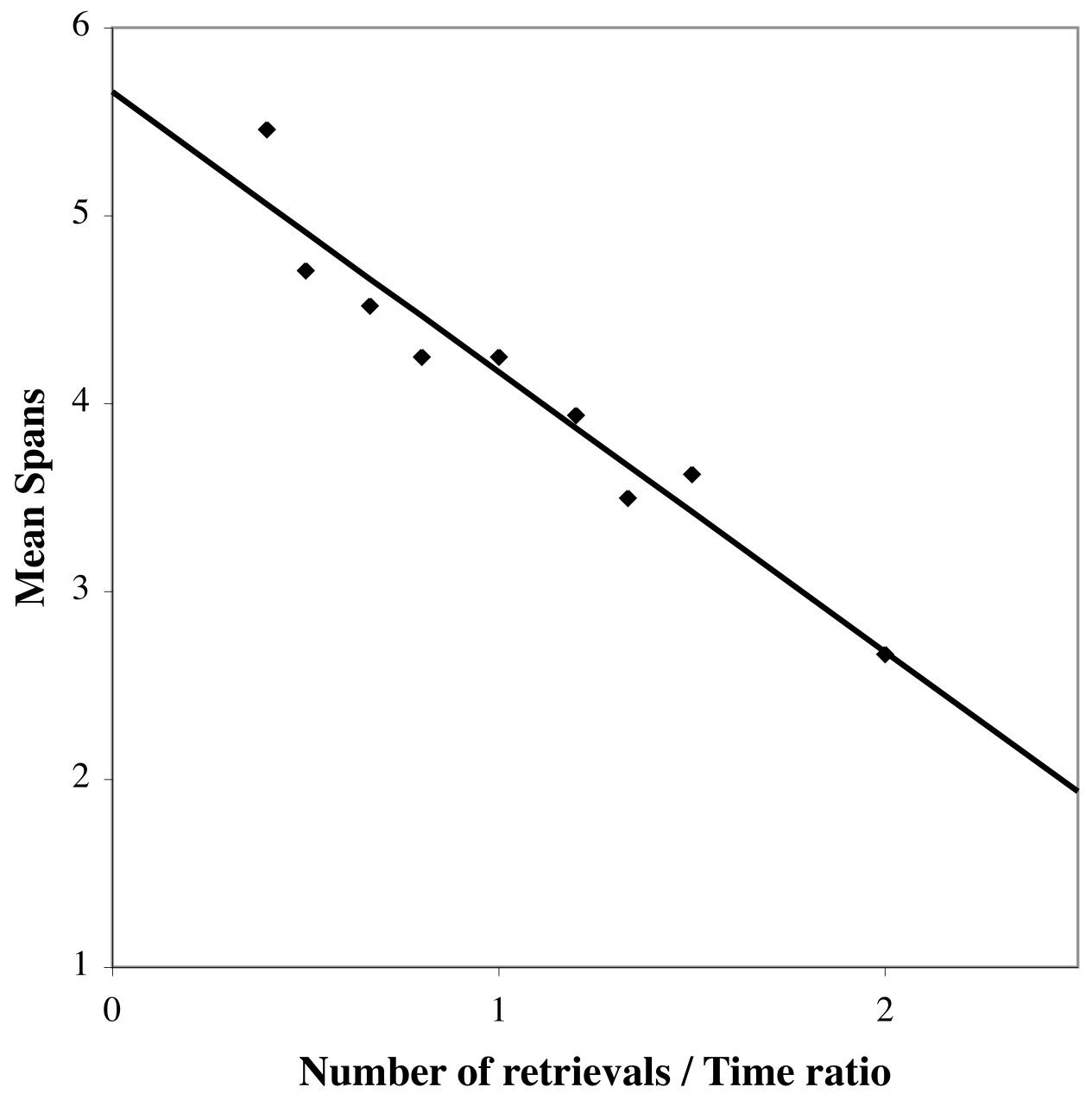

Figure 1 


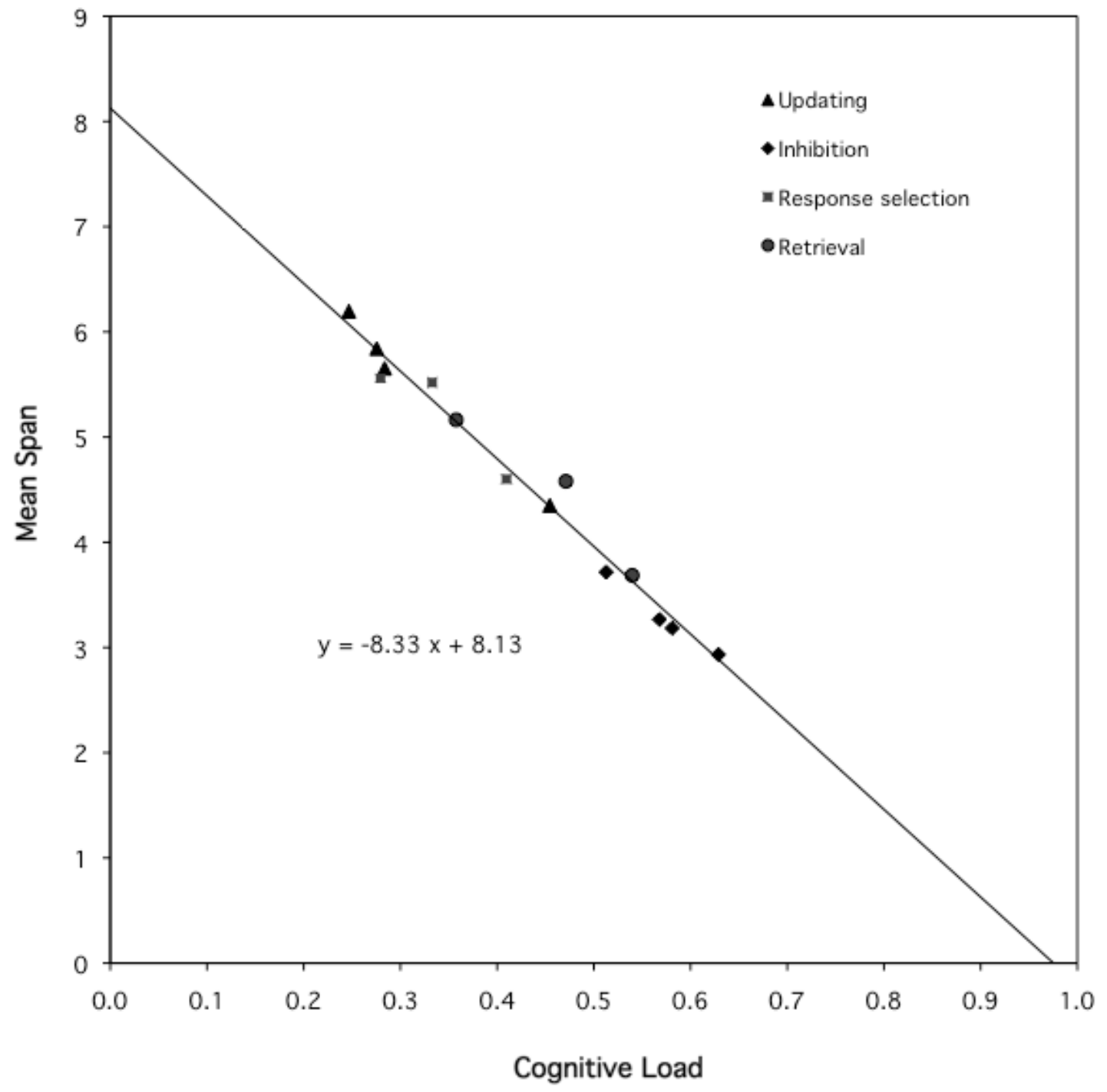

Figure 2 


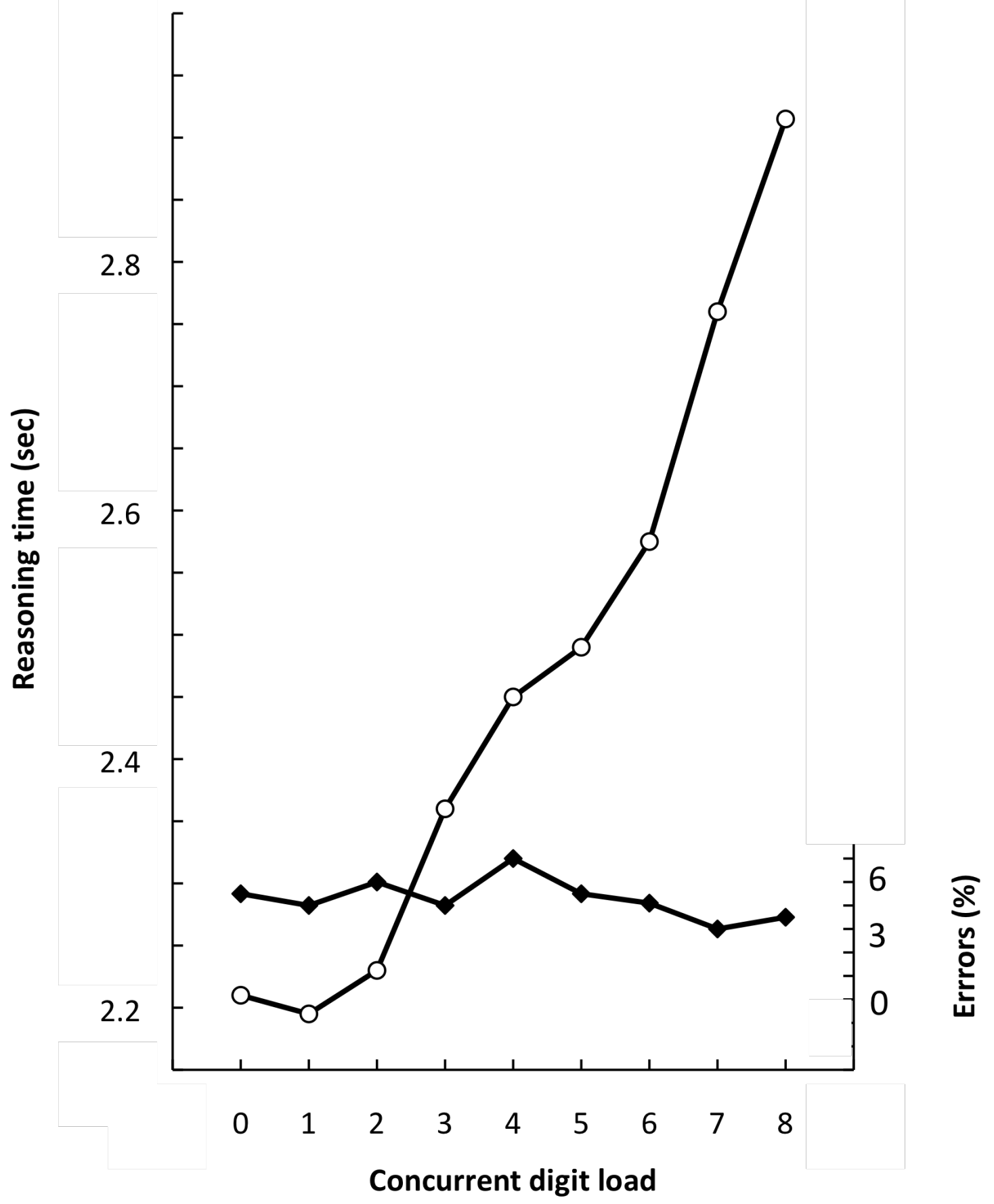

Figure 3 